\title{
Actividad Antibacteriana de una Resina Ortodóntica Modificada con Diferentes Concentraciones de Nanopartículas de Óxido de Cobre
}

\author{
Antibacterial Activity of an Orthodontic Resin Modified with \\ Different Concentration of Copper Oxide Nanoparticles
}

Marco Antonio Sánchez-Tito

SÁNCHEZ-TITO, M. A. Actividad antibacteriana de una resina ortodóntica modificada con diferentes concentraciones de nanopartículas de óxido de cobre. Int. J. Odontostomat., 15(3):595-601, 2021.

RESUMEN: El objetivo del estudio fue evaluar la actividad antibacteriana de un cemento ortodóntico modificado con diferentes concentraciones de nanopartículas de óxido de cobre (NPOCu) frente a Streptococcus mutans y Lactobacillus acidophilus. Se prepararon discos de resina conteniendo concentraciones de $1 \%, 0,5 \%$, 0,1 \% y 0,05 \% de NPOCu. Los discos fueron sometidos a un ensayo microbiológico de contacto directo, sobre placas Petri inoculadas con suspensiones de las cepas. Los halos de inhibición se midieron con un compás Vernier. Adicionalmente se realizó el conteo de las unidades formadoras de colonias (UFC).Se usaron discos de resina sin agregado de NPOCu como grupo control. Los resultados mostraron que las concentraciones de $1 \%, 0,5 \%, 0,1 \%$ y 0,05 \% de NPOCu fueron efectivas para inhibir el

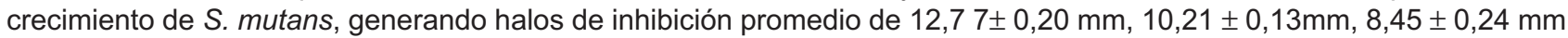
y $6,22 \pm 0,37 \mathrm{~mm}$, respectivamente. Mientras que las concentraciones del $1 \%, 0,5 \%$ y $0,1 \%$ inhibieron el crecimiento de $L$. acidophilus con halos de inhibición de $11,95 \pm 0,17 \mathrm{~mm}, 9,24 \pm 0,14 \mathrm{~mm}$ y 6,21 $\pm 0,20 \mathrm{~mm}$. Se observaron diferencias significativas cuando se comparó el efecto de las concentraciones sobre ambas cepas $(p<0,05)$. Adicionalmente, todas las concentraciones de NPOCu mostraron un conteo de UFC significativamente menor que el grupo control, para ambas cepas $(p<0,05)$. Como conclusión podemos señalar que la incorporación de diferentes concentraciones de nanopartículas de óxido de cobre en un cemento ortodóntico posee un importante efecto antibacteriano frente a S. mutans y L. acidophilus.

PALABRAS CLAVE: agente antibacteriano, ortodoncia, nanopartículas, cobre.

\section{INTRODUCCIÓN}

Las lesiones de mancha blanca (LMB) son uno de los principales efectos adversos del tratamiento ortodóntico y pueden afectar a más del $70 \%$ de estos pacientes (Sundararaj et al., 2015; Eltayeb et al., 2017; Khoroushi \& Kachuie, 2017). La ocurrencia de estas lesiones está asociada principalmente a una deficiente higiene oral, el aumento en el consumo de alimentos y bebidas acidogénicas, tratamientos de duración prolongada y tratamientos a edades tempranas (Sundararaj et al., Srivastava et al., 2013). Si bien las LMB pueden afectar cualquier diente, se ha reportado que son más frecuentes en los incisivos laterales, caninos y premolares (Sagarika et al., 2012; Eltayeb et al.); por otro lado, cualquier parte de la superficie de los dientes puede verse afectada; sin embargo, se pre- senta de manera frecuente en las regiones cervical adyacente a los brackets.

La formación de las LMB se produce debido a un incremento en la colonización de bacterias acidogénicas, consecuentemente sus subproductos pueden reducir el $\mathrm{pH}$ del medio bucal a valores críticos, incluso inferiores a 5,5 (Sangamesha et al., 2020. Estas condiciones promueven el ingreso de los ácidos a través de la subsuperficie del esmalte, donde se disocian y facilitan la degradación de los cristales de hidroxiapatita, formando la lesión inicial de desmineralización, que debido a la refracción de la luz se observan como lesiones blanquecinas y opacas (Velazquez-Enriquez et al., 2012; Jing et al., 2019). 
En los pacientes con tratamiento ortodóntico fijo, que no siguen un régimen adecuado de higiene oral, la aparición de las LMB puede ocurrir tan pronto como luego de un mes de iniciado el tratamiento (Lombardo et al., 2013).

La principal estrategia para prevenir su aparición es promover un régimen correcto de higiene oral y se ha recomendado el uso de aplicaciones tópicas de flúor o barnices, el uso de pasta dentales o enjuagues bucales con agentes antibacterianos, todas estas estrategias han demostrado ser efectivas para prevenir la aparición de las LMB, sin embargo, son dependientes de la colaboración del paciente, lo que puede disminuir su efectividad (Hoffman et al., 2015; Heravi et al., 2018; Farzanegan et al., 2019; Hu et al., 2019; Kozak et al., 2020).

Con el objetivo de eliminar el factor de colaboración del paciente, diversos reportes han propuesto la incorporación de agentes antibacterianos en los cementos ortodónticos como sales de amonio cuaternario, cloruro de benzalconio, nanopartículas sintetizadas a partir de metales o productos naturales (Reddy et al., 2016; Sodagar et al., 2017; Torres-Garcia et al., 2019; Yassaei et al., 2020). Todas estas estrategias han sido efectivas en controlar e inhibir el crecimiento de bacterias asociadas con la formación de las LMB.

El uso de las nanopartículas metálicas tiene un particular interés en el campo de la odontología y se sabe que sus propiedades antibacterianas están asociadas la actividad de los iones libres sobre la membrana de los microorganismos, permitiendo una degradación directa y promoviendo su ingreso al citoplasma donde actúan a distinto nivel, promoviendo la muerte celular (Vimbela et al., 2017; Song \& Ge, 2019; JassoRuiz et al., 2020). Las nanopartículas de óxido de cobre (NPOCu), han sido incorporadas a diversos materiales dentales como resinas compuestas, bases acrílicas, acrílicos de placas removibles, entre otros, con la inten- ción de inhibir el crecimiento bacteriano. Además, se sabe que el agregado de estas nanopartículas no afecta las propiedades físicas y mecánicas de estos materiales cuando se emplean en concentraciones bajas pero suficiente para conservar sus propiedades antibacterianas (Amiri et al., 2017; Yassaei et al.; Toodehzaeim et al.; Ferrando-Magraner et al., 2020).

El objetivo del estudio fue evaluar la actividad antibacteriana de un cemento ortodóntico modificado con diferentes concentraciones de NPOCu frente a Streptococcus mutans y Lactobacillus acidophilus.

\section{MATERIAL Y MÉTODO}

Se diseñó una investigación experimental in vitro, que fue aprobada por el Comité de Ética en Investigación de la Universidad. El cálculo de las repeticiones para los ensayos microbiológicos se realizó empleando el programa G Power. Considerando un nivel de confianza del $95 \%$ y un poder estadístico del $80 \%$.

\section{Preparación de la resina modificada con NPOCu.} Se empleó una resina ortodóntica comercial (Transbond XT,3M Unitek, Monrovia, CA, EE.UU.) que fue mezclada con nanopartículas de óxido de cobre (US Research Nanomaterials, Inc. Houston, TX, EE.UU.), los materiales y sus composiciones pueden observarse en la Tabla I. La preparación se realizó empleando una espátula plástica sobre una platina de vidrio, en un ambiente oscuro. Las resinas fueron mezcladas por dos minutos de manera consistente para asegurar una correcta distribución de las nanopartículas en la matriz de la resina. En la Tabla II se presentan las cantidades de resina y de las nanopartículas para obtener las concentraciones $1 \%$; $0,5 \% ; 0,1 \%$ y $0,05 \%$ que fueron pesadas en una balanza analítica para asegurar la precisión.

Tabla I. Materiales empleados en el estudio.

\begin{tabular}{llll}
\hline Material y fabricante & Tipo de material & \multicolumn{1}{c}{ Composición } & No. Lote \\
\hline Transbond XT (3M Unitek, & Adhesivo en pasta & Cuarzo tratado con silano, bisfenol A diglicidil & N976370 \\
Monrovia, CA, EE.UU.) & fotopolimerizable & $\begin{array}{l}\text { éter dimetacrilato, bisfenol A bis (2-hidroxietil } \\
\text { éter) dimetacrilato, sílice tratada con silano, }\end{array}$ & hexafluouofosfato de difeniliodonio.
\end{tabular}

Nanopartículas de óxido de Nano-polvo cobre (US Research

Nanomaterials, Inc. Houston, TX, EE.UU.)
Tamaño promedio de la nanopartícula: $40 \mathrm{~nm}$, pureza: $99.99 \%$.
Stock\#: US1038

CAS\#: 7440-22-4 
Tabla II. Composición de las resinas experimentales.

\begin{tabular}{ll} 
Concentración & Resina (peso) $/$ AgNPs (peso) \\
\hline $1 \%$ NPOCu & $3 \mathrm{~g}$ resina $+0.03 \mathrm{~g} \mathrm{NPOCu}$ \\
$0,5 \% \mathrm{NPOCu}$ & $3 \mathrm{~g}$ resina $+0.015 \mathrm{~g} \mathrm{NPOCu}$ \\
$0,1 \% \mathrm{NPOCu}$ & $3 \mathrm{~g}$ resina $+0.003 \mathrm{~g} \mathrm{NPOCu}$ \\
$0,05 \% \mathrm{NPOCu}$ & $3 \mathrm{~g}$ resina $+0.0015 \mathrm{~g} \mathrm{NPOCu}$ \\
$0 \% \mathrm{NPOCu}$ & $3 \mathrm{~g}$ resina sin agregado de NPOCu
\end{tabular}

Preparación de los discos de resina. Se prepararon 45 discos de resina (9 discos por grupo). Los discos se realizaron con la ayuda de un molde de plástico de 5 $\mathrm{mm}$ de diámetro y $1 \mathrm{~mm}$ de grosor. La resina fue cubierta con una cinta de celuloide y fue polimerizada por 20 segundos por cada lado con una Unidad Led (Elipar Deep Cure-L, 3M, St. Paul, MN, USA). Los excesos de resina fueron pulidos con discos y esterilizados con irradiación ultravioleta por 15 min.

Suspensión bacteriana. Se emplearon cepas de Streptococcus mutans ATCC $₫ 25175^{\mathrm{TM}}$ y Lacto bacillus acidophilus ATCC $₫ 4356^{\mathrm{TM}}$ (American Type Culture Collection, Manassas, VA, EE.UU.). S. mutans se cultivó en caldo cerebro-corazón (BHI), mientras que $L$. acidophilus en caldo MRS (Man, Rogosa y Sharpe), ambas suspensiones fueron incubadas a $37^{\circ} \mathrm{C}$ por 7 horas hasta alcanzar la fase logarítmica de crecimiento bacteriano. Se transfirieron alícuotas a tubos conteniendo suero fisiológico y se ajustó la turbidez a la escala 0,5 de McFarland. Los medios de cultivo fueron adquiridos de Liofilchem $\AA$ (Rosetodegli Abruzzi, TE, Italia).

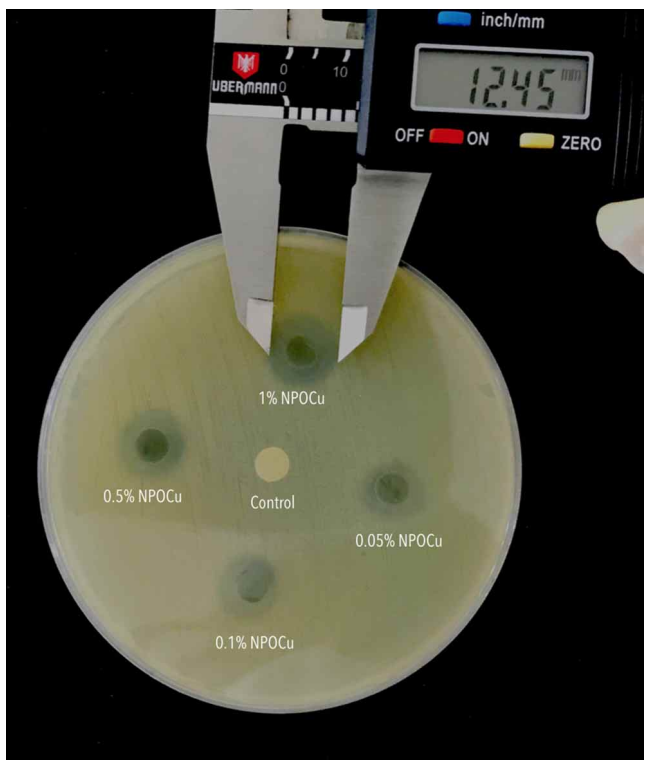

Fig. 1. Medición de los halos de inhibición producidos por las NPOCu sobre S. mutans.
Ensayo de contacto directo. Se transfirieron $50 \mu \mathrm{L}$ de las suspensiones bacterianas en placas Petri conteniendo agar cerebro-corazón (BHA) y agar MRS, para S. mutans y L. acidophilus, respectivamente. Se emplearon 5 placas por cada cepa donde se colocaron en cada una de ellas uno de los discos de resina de cada concentración y el disco de resina sin modificar, considerando una distancia mínima de $2 \mathrm{~cm}$ entre los discos. En el caso de $S$. mutans las placas fueron incubadas por $48 \mathrm{~h}$ en una jarra de anaerobiosis empleando en sistema GaspakTM EZ Campy Container System para generar un ambiente de microaerofilia, $L$. acidophilus se incubó en una atmósfera de $5 \%$ CO2. Enseguida, la formación de los halos de inhibición de crecimiento bacteriano se midió con un compás Vernier digital (Ubermann $®$, Chile) (Fig. 1).

Cuantificación de las Unidades formadoras de coIonia (UFC). Para registrar las UFC se realizó un ensayo de dilución en placas de 96 pozos. Se colocaron $200 \mu \mathrm{L}$ de caldo $\mathrm{BHI}$ enriquecido con $1 \%$ de sucrosa en los pozos de las columnas $1,3,5,7,11$ y 12 de las filas A, C, E y G. Se agregaron $15 \mu \mathrm{L}$ de la suspensión de $S$. mutans y se colocó un disco de las resinas experimentales. El esquema de la distribución de los discos de resina en los pozos de la placa se puede observar en la Figura 2. Las placas fueron incubadas a $37^{\circ} \mathrm{C}$ por $24 \mathrm{~h}$. Posteriormente se realizaron diluciones a una concentración de $10^{-6}$ empleando $100 \mu \mathrm{L}$ de la suspensión de cada pozo con $900 \mu \mathrm{L}$ de solución $0,5 \% \quad 0,1 \% \quad 0,05 \% \quad 0 \%$ (Control) $\quad C_{+} \quad$ c-

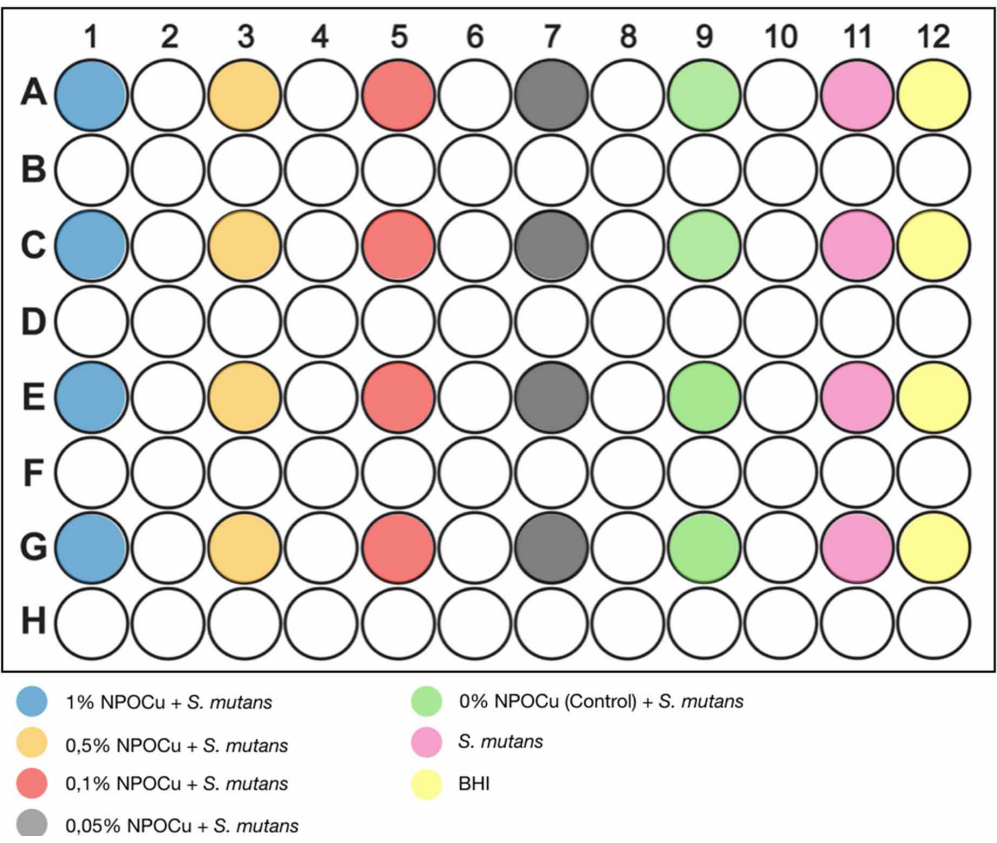

Fig. 2. Esquema de la distribución de las resinas modificadas con NPOCu en la microplaca de 96 pozos. 
salina. Se empleó $50 \mu \mathrm{L}$ de la dilución final que fueron sembrados en placas Petri conteniendo Agar BHA. Las placas fueron incubadas a $37^{\circ} \mathrm{C}$ por $48 \mathrm{~h}$ y el número de UFC fueron contadas y transformadas a la expresión logarítmica por mililitro (Log UFC/mL). El ensayo se repitió para $L$. acidophilus empleando caldo y agar MRS como medios de cultivo.

Análisis estadístico. Los datos fueron analizados con el programa SPSS en su versión 23.0 para Mac OS (SPSS Inc., IBM, NY, EE.UU.). La normalidad de la distribución de los datos fue analizada con la prueba de Shapiro-Wilk. Se empleó la prueba de análisis de la varianza (ANOVA) para comparar el efecto antibacteriano de las resinas con las concentraciones de NPOCu. Las comparaciones intra-grupos se realizó con la prueba de Tukey. Se estableció un nivel de significancia del $5 \%$ para las pruebas.

\section{RESULTADOS}

La Tabla III muestra los resultados de la prueba ANOVA seguida de la prueba post hoc de Tukey del estudio de difusión de discos, donde se observa que los discos de resina con todos los agregados de NPOCu presentaron inhibición del crecimiento

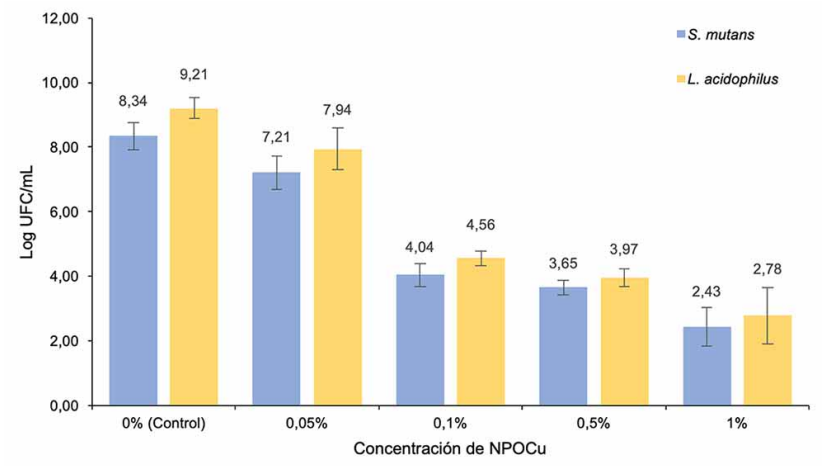

Fig. 3. Unidades formadoras de colonia (Log UFC/mL) de $S$. mutans y $L$. acidophilus. bacteriano sobre $S$. mutans y a la vez estos resultados fueron significativamente diferentes entre sí $(p<0,05)$. En el caso de $L$. acidophiluslas concentraciones con agregados de NPCOu de $1 \%, 0,5 \%$ y $0,1 \%$ presentaron efecto antibacteriano, mientras que la concentración de $0,05 \%$ no generó inhibición del crecimiento sobre $L$. acidophilus. Cuando se compararon los efectos antibacterianos de las concentraciones sobre ambas cepas, los halos de inhibición generados por los discos de resina de las concentraciones del $1 \%, 0,5 \%$ y $0,1 \%$ fueron estadísticamente diferentes $(p<0,05)$.

Todas las resinas modificadas con las distintas concentraciones de NPOCu mostraron un conteo de UFC significativamente menor que el grupo control $(p<0,05)$ para $S$. mutans y $L$. acidophilus. Se observó una relación inversamente proporcional entre la disminución del conteo de UFC y el incremento de la concentración de NPOCu contenida en los discos de resina.

\section{DISCUSIÓN}

Las LMB continúan siendo uno de los principales problemas asociados al tratamiento de ortodoncia con aparatos fijos y están relacionadas a situaciones como la pobre higiene oral, el aumento en el consumo de alimentos acidogénicos tiempos de tratamiento prolongados y la mala adhesión de los pacientes al tratamiento (Eltayeb et al., Sundararaj et al., Srivastava et al.). De todas las estrategias que se describen en la literatura para la prevención y control de estas alteraciones, recientemente, el uso de agentes antibacterianos incluidos en las resinas ortodónticas ha despertado en interés de los investigadores, proponiendo el uso de derivados de las sales de amonio cuaternario, cloruro de benzalconio, hidroxiapatita, nanopartículas metálicas y de productos naturales, dentro de ellas las NPOCu (Reddy et al.; Sodagar et al.; Torres-Garcia et al.; Yassaei et al.).

Tabla III. Medias de los halos de inhibición $(\mathrm{mm})$ generados por los cementos modificados con NPOCu sobre las cepas estudiadas.

\begin{tabular}{lcccccc}
\hline \multicolumn{5}{c}{ Porcentajes NPOCu } \\
\hline Microorganismo & $\begin{array}{c}1 \% \text { NPOCu } \\
\text { (Media } \pm D E)\end{array}$ & $\begin{array}{c}0,5 \% \text { NPOCu } \\
\text { (Media } \pm \text { DE) }\end{array}$ & $\begin{array}{c}0,1 \% \text { NPOCu } \\
\text { (Media } \pm D E)\end{array}$ & $\begin{array}{c}0,05 \% \text { NPOCu } \\
\text { (Media } \pm D E)\end{array}$ & $\begin{array}{c}0 \% \text { NPOCu } \\
\text { (control) }\end{array}$ & $\begin{array}{c}\text { Valor- } \\
P\end{array}$ \\
\hline S. mutans & $12,77 \pm 0,20^{\mathrm{Aa}}$ & $10,21 \pm 0,13^{\mathrm{Ba}}$ & $8,45 \pm 0,24 \mathrm{C}^{\mathrm{a}}$ & $6,22 \pm 0,37 \mathrm{D}$ & 0,00 & 0,000 \\
L. acidophilus & $11,95 \pm 0,17^{\mathrm{Ab}}$ & $9,24 \pm 0,14^{\mathrm{Bb}}$ & $6,21 \pm 0,20 \mathrm{c}^{\mathrm{b}}$ & 0,00 & 0,00 & 0,000 \\
\hline
\end{tabular}

DE: Desviación estándar. Las letras mayúsculas en superíndice indica diferencias significativas entre las filas $(p<0,05)$, mientras que las letras minúsculas en superíndice representan diferencias significativas entre las columnas $(p<0,05)$. 
El objetivo del estudio fue evaluar el efecto antibacteriano de un cemento ortodóntico modificado con diferentes concentraciones de NPOCu frente a $S$. mutans y L.acidophilus. Los resultados mostraron que todas las concentraciones fueron efectivas para inhibir el crecimiento de $S$. mutans, mientras que para $L$. acidophilus las concentraciones del $1 \%, 0,5 \%$ y 0,1 $\%$ de NPOCu fueron efectivas para inhibir su crecimiento, la concentración del $0,05 \%$ al igual que el cemento sin agregado de NPOCu no generaron halos de inhibición en la prueba de contacto directo.

Se ha descrito que la actividad antibacteriana de las NPOCu está relacionada ala razón entre la superficie/volumen, lo que permite que estas nanopartículas penetren las membranas bacterianas, en donde se liberan iones de $\mathrm{Cu} 2+$, además se sabe que se produce una atracción electrostática entre la carga negativa de la pared celular bacteriana, permitiendo el ingreso de más iones de $\mathrm{Cu} 2+$ al citoplasma, produciendo una alteración en las enzimas respiratorias, interfiriendo también en la replicación del ADN, lo que evita la replicación bacteriana y en consecuencia promueve la muerte celular (Vimbela et al.; Song \& Ge; Jasso-Ruiz et al.).

El tamaño de las nanopartículas está relacionado con la actividad antibacteriana, nanopartículas más pequeñas presentan mayor efecto antibacteriano, aunque también mayores efectos citotóxicos (Vimbela et al.; Song \& Ge). En este estudio las NPOCu tuvieron un tamaño de $40 \mathrm{~nm}$, los estudios previos en donde se incorporó este tipo de nanopartículas en cementos ortodónticos han demostrado una importante actividad antibacteriana frente a diversas especies bacterianas, aunque no se reporta el tamaño de las nanopartículas empleadas. Toodehzaeim et al. [MOU3] encontraron que el agregado de NPOCu en concentraciones de $0,01,0,5$ y $1 \%$ presentó un efecto antibacteriano frente a $S$. mutans con la formación de zonas de inhibición entre 8,36 y $9,38 \mathrm{~mm}$, no existiendo diferencia entre las concentraciones empleadas. Yassaei et al. reportaron resultados similares donde agregados de $1 \%$ y $0,5 \%$ de NPOCu en una resina ortodóntica generó halos de inhibición de 7,5 y $8 \mathrm{~mm}$ sobre el crecimiento de $S$. mutans, aunque estos resultados no se mantuvieron en la evaluación de 15 a 30 días. Estos resultados fueron menores que los encontrados en nuestro estudio, donde la concentración de 1 $\%$ y $0,5 \%$ generaron halos de inhibición sobre la misma bacteria de 12,77 y $10,21 \mathrm{~mm}$, respectivamente.

Adicionalmente en este estudio se realizó el análisis de las UFC, todas las concentraciones de
NPOCu mostraron un conteo significativamente menor de UFC que las generadas en el grupo control, para las dos cepas estudiadas. No existen reportes que empleen esta metodología para evaluar el efecto del agregado de las NPOCu en resinas ortodónticas, sin embargo, se ha utilizado con nanopartículas de plata, demostrando ser una metodología útil para evaluar el efecto antibacteriano frente a diversas bacterias cariogénicas (Sodagar et al.; Twomley et al., 2019). Los resultados mostraron una relación inversamente proporcional entre la disminución del conteo de UFC y el incremento de la concentración de NPOCu.

En la literatura se aborda las propiedades antibacterianas de las NPOCu en asociación con diversos materiales de uso ortodóntico, incluso como revestimiento de brackets, al respecto Ramazanzadeh et al. (2015) demostraron que el revestimiento de brackets metálicos con NPOCu evita la colonización de $S$. mutans y otras bacterias cariogénicas. ArguetaFigueroa et al. (2015) investigaron los efectos antibacterianos sobre $S$. mutans de agregados de 0,0100 \%, 0,0075\% y 0,0050 \% de naonpartículas de cobre a un adhesivo ortodóntico, encontrando que la concentración al 0,0100 \% fue la única que presentó propiedades antibacterianas frente a $S$. mutans similares a los de la clorhexidina.

La concentración más alta de NPOCu empleada en este estudio fue de $1 \%$, se sabe que concentraciones mayores de diversas nanopartículas empleadas en ensayos similares pueden ser citotóxicas en estudios in vitro, además tienen un efecto negativo sobre el color de la resina, lo que afecta las propiedades de polimerización y en consecuencia las propiedades mecánicas de las mismas (de Almeida et al., 2018; Yassaei et al.).

Las estrategias que buscan incorporar nanopartículas con actividad antibacteriana tienen el potencial de eliminar el factor de colaboración del paciente, que afecta la eficacia de las intervenciones tradicionales como el uso de enjuagues, barnices o colutorios para el control de la placa bacteriana. Esta investigación presenta algunas limitaciones, como por ejemplo el diseño in vitro enfocado a la actividad antibacteriana no permite extrapolar estos resultados al comportamiento clínico que podría tener la incorporación de las NPOCu en cementos ortodónticos. Por lo que es necesario el desarrollo de estudios para valorar la toxicidad de estos productos y diseñar ensayos clínicos que permitan esclarecer algunos aspectos respecto a su potencial empleo clínico. 


\section{CONCLUSIONES}

La incorporación de nanopartículas de óxido de cobre en concentraciones de $1 \%, 0,5 \%, 0,1 \%$ y $0,05 \%$ en un cemento ortodóntico demostró un importante efecto antibacteriano frente a S. mutans, mientras que las concentraciones de $1 \%, 0,5 \%$ y $0,1 \%$ fueron efectivas frente a $L$. acidophilus.

SÁNCHEZ-TITO, M. A. Antibacterial activity of an orthodontic resin modified with different concentration of copper oxide nanoparticles. Int. J. Odontostomat., 15(3):595-601, 2021.

ABSTRACT: The objective of the study was to evaluate the antibacterial activity of an orthodontic cement modified with different concentrations of copper oxide nanoparticles (CuONP) against Streptococcus mutans and Lactobacillus acidophilus. Resin discs containing concentrations of $1 \%, 0.5 \%, 0.01 \%$ and $0.05 \%$ of CuONP were prepared. The disks were subjected to a direct contact microbiological test on Petri dishes inoculated with suspensions of the strains. Inhibition halos were measured with a Vernier compass. Additionally, the colony forming units (CFU) were counted. Resin discs without CuONP were used as a control group. The results showed that the concentrations of $1 \%, 0.5 \%, 0.01 \%$ and $0.05 \%$ of CuONP were effective in inhibiting the growth of $S$. mutans, generating average inhibition halos of $12.77 \pm 0.20 \mathrm{~mm}, 10.21 \pm 0.13 \mathrm{~mm}, 8.45 \pm$ $0.24 \mathrm{~mm}$ and $6.22 \pm 0.37 \mathrm{~mm}$ respectively. While the concentrations of $1 \%, 0.5 \%$ and $0.01 \%$ inhibited the growth of $L$. acidophilus with inhibition halos of $11.95 \pm 0.17 \mathrm{~mm}$, $9.24 \pm 0.14 \mathrm{~mm}$ and $6.21 \pm 0.20 \mathrm{~mm}$. Significant differences were observed when the effect of the concentrations on both strains was compared $(p<0.05)$. Additionally, all CuONP concentrations showed a CFU count significantly lower than the control group, for both strains $(p<0.05)$. In conclusion, we can point out that the incorporation of different concentrations of copper oxide nanoparticles in orthodontic cement has an important antibacterial effect against $S$. mutans and $L$. acidophilus.

KEY WORDS: antibacterial agent, orthodontics, nanoparticles, copper.

\section{REFERENCIAS BIBLIOGRÁFICAS}

Amiri, M.; Etemadifar, Z.; Daneshkazemi, A. \& Nateghi, M. Antimicrobial effect of copper oxide nanoparticles on some oral bacteria and candida species. J. Dent. Biomater., 4(1):347-52, 2017.

de Almeida, C. M.; da Rosa, W. L. O.; Meereis, C. T. W.; de Almeida, S. M.; Ribeiro, J. S.; da Silva, A. F. \& Lund, R. G. Efficacy of antimicrobial agents incorporated in orthodontic bonding systems: a systematic review and meta-analysis. J. Orthod., 45(2):79-93, 2018.
Farzanegan, F.; Morteza-Saadat-Mostafavi, S.; Ameri, H. \& Khaki $\mathrm{H}$. Effects of fluoride versus amorphous calcium phosphate solutions on enamel microhardness of white spot lesions: An invitro study. J. Clin. Exp. Dent., 11(3):e219-24, 2019.

Ferrando-Magraner, E.; Bellot-Arcís, C.; Paredes-Gallardo, V.; Almerich-Silla, J. M.; García-Sanz, V.; Fernández-Alonso, M. \& Montiel-Company, J. M. Antibacterial properties of nanoparticles in dental restorative materials. A systematic review and metaanalysis. Medicina (Kaunas), 56(2):55, 2020.

Heravi, F.; Ahrari, F. \& Tanbakuchi, B. Effectiveness of MI Paste Plus and Remin Pro on remineralization and color improvement of postorthodontic white spot lesions. Dent. Res. J. (Isfahan), 15(2):95-103, 2018.

Hoffman, D. A.; Clark, A. E.; Rody, W. J. Jr.; McGorray, S. P. \& Wheeler, T. T. A prospective randomized clinical trial into the capacity of a toothpaste containing NovaMin to prevent white spot lesions and gingivitis during orthodontic treatment. Prog. Orthod., 16(1):25, 2015.

Hu, H.; Feng, C.; Jiang, Z.; Wang, L.; Shrestha, S.; Su, X.; Shu, Y.; Ge, L.; Lai, W.; Hua, F. \& Long, H. Effectiveness of remineralising agents in prevention and treatment of orthodontically induced white spot lesions: a protocol for a systematic review incorporating network meta-analysis. Syst. Rev., 8(1):339, 2019.

Jasso-Ruiz, I.; Velazquez-Enriquez, U.; Scougall-Vilchis, R. J.; Morales-Luckie, R. A.; Sawada, T. \& Yamaguchi, R. Silver nanoparticles in orthodontics, a new alternative in bacterial inhibition: in vitro study. Prog. Orthod., 21(1):24, 2020.

Jing, D.; Hao, J.; Shen, Y.; Tang, G.; Lei, L. \& Zhao, Z. Effect of fixed orthodontic treatment on oral microbiota and salivary proteins. Exp. Ther. Med., 17(5):4237-43, 2019.

Khoroushi, M. \& Kachuie, M. Prevention and treatment of white spot lesions in orthodontic patients. Contemp. Clin. Dent., 8(1):1119, 2017.

Kozak, U.; Se kowska, A. \& Cha?as, R. The effect of regime oralhygiene intervention on the incidence of new white spot lesions in teenagers treated with fixed orthodontic appliances. Int. J. Environ. Res. Public. Health, 17(24):9460, 2020.

Lombardo, L.; Ortan, Y. Ö.; Gorgun, Ö.; Panza, C.; Scuzzo, G. \& Siciliani, G. Changes in the oral environment after placement of lingual and labial orthodontic appliances. Prog. Orthod., 14(1):28, 2013.

Ramazanzadeh, B.; Jahanbin, A.; Yaghoubi, M.; Shahtahmassbi, N.; Ghazvini, K.; Shakeri, M. \& Shafaee, H. Comparison of antibacterial effects of $\mathrm{ZnO}$ and $\mathrm{CuO}$ nanoparticles coated brackets against Streptococcus mutans. J. Dent. (Shiraz), 16(3):200-5, 2015.

Reddy, A. K.; Kambalyal, P. B.; Patil, S. R.; Vankhre, M.; Khan, M. Y. \& Kumar, T. R. Comparative evaluation and influence on shear bond strength of incorporating silver, zinc oxide, and titanium dioxide nanoparticles in orthodontic adhesive. J. Orthod. Sci., 5(4):127-31, 2016.

Sagarika, N.; Suchindran, S.; Loganathan, S. \& Gopikrishna, V. Prevalence of white spot lesion in a section of Indian population undergoing fixed orthodontic treatment: An in vivo assessment using the visual International Caries Detection and Assessment System II criteria. J. Conserv. Dent., 15(2):104-8, 2012.

Sodagar, A.; Akhoundi, M. S. A.; Bahador, A.; Jalali, Y. F.; Behzadi, Z.; Elhaminejad, F. \& Mirhashemi, A. H. Effect of TiO2 nanoparticles incorporation on antibacterial properties and shear bond strength of dental composite used in Orthodontics. Dental Press J. Orthod., 22(5):67-74, 2017.

Song, W. \& Ge, S. Application of antimicrobial nanoparticles in dentistry. Molecules, 24(6):1033, 2019.

Srivastava, K.; Tikku, T.; Khanna, R. \&Sachan, K. Risk factors and management of white spot lesions in orthodontics. J. Orthod. Sci., 2(2):43-9, 2013 
Sundararaj, D.; Venkatachalapathy, S.; Tandon, A. \& Pereira, A. Critical evaluation of incidence and prevalence of white spot lesions during fixed orthodontic appliance treatment: A metaanalysis. J. Int. Soc. Prev. Community Dent., 5(6):433-9, 2015.

Torres-Garcia, M. L.; Llavore, L. D.; Bungay, A.; Sarol, J. D. Jr.; Pineda, R. R. \& Peñas, K. D. Benzalkonium chloride in an orthodontic adhesive: Its effect on rat enamel demineralization using color-based image analysis. Am. J. Orthod. Dentofacial Orthop., 155(1):88-97, 2019.

Twomley, J.; Wang, Y.; Wen, Z.; Yu, Q.; Ballard, R.; Armbruster, P. \& $\mathrm{Xu}, \mathrm{X}$. Formulation and characterization of antibacterial orthodontic adhesive. Dental Press J. Orthod., 24(4):73-9, 2019.

Velazquez-Enriquez, U.; Scougall-Vilchis, R. J.; Contreras-Bulnes, R.; Flores-Estrada, J.; Uematsu, S. \& Yamaguchi, R. Quantitative analysis of $S$. mutans and $S$. sobrinus cultivated independently and adhered to polished orthodontic composite resins. J. Appl. Oral Sci., 20(5):544-9, 2012.

Vimbela, G. V.; Ngo, S. M.; Fraze, C.; Yang, L. \& Stout, D. A. Antibacterial properties and toxicity from metallic nanomaterials. Int. J. Nanomedicine, 12:3941-65, 2017.

Yassaei, S.; Nasr, A.; Zandi, H. \& Motallaei, M. N. Comparison of antibacterial effects of orthodontic composites containing different nanoparticles on Streptococcus mutans at different times. Dental Press J.Orthod., 25(2):52-60, 2020.
Dirección para correspondencia:

Marco A. Sánchez-Tito

Facultad de Ciencias de la Salud

Universidad Privada de Tacna

Av. Jorge Basadre Grohmann s/n

Tacna

PERÚ

E-mail: marcosanchez2183@gmail.com 